

\section{Corriente principal}

Scopus es una de las bases bibliográficas académicas de mayor prestigio mundial, creada por Elsevier. Tiene una amplia cobertura de publicaciones en ciencias sociales y humanas e integra el conocimiento de distintos continentes. Dada su amplia cobertura, tanto geográfica como temática, es ideal para ser utilizada para revisiones bibliográficas, incluyendo las revisiones sistemáticas (systematic reviews, como las denominan otros autores, comprehensive literary reviews) en cualquier área.

Por otra parte, Web of Science (WoS) (antes conocida como ISI, fue creada por el Information Science Institute) creada por Eugene Garfield como fuente para determinar el factor de impacto en la evaluación de la producción científica. En los últimos años WOS ha mejorado sus políticas de integración, sin embargo, continua con una baja representación de las ciencias sociales y humanas, así como la producción de África y América del Sur.

En este contexto, el boletín blibliométrico de la USTA presenta la participación de la Universidad en estas importantes bases bibliográficas durante el periodo comprendido entre 2004 y 2018. Es importante destacar el aumento significativo de la producción en revistas indexadas Scopus, Wos, que pasa de 3 publicación en 2004; a 124 en 2018 en Scopus y de 1 publicaciones en WOS a 102 en el mismo periodo de tiempo. Así mismo, en 2018 los artículos publicados por autores de la USTA alcanzan un valor promedio de citas superior a 1000. Por otra parte, se destacan los autores USTA en Scopus y Wos con más publicaciones y con más citaciones de la Universidad, así como los países del mundo con los que colaboran nuestros investigadores, las áreas temáticas y los tipos documentales de las publicaciones.

Finalmente, invitamos a todos los investigadores y docentes a utilizar esta importante herramienta que nos ofrece el Observatorio de Bibliometría y Cienciometría de la USTA y a participar activamente de las estrategias trazadas por la Vicerrectoría Académica General a través de la División de Investigación e innovación, con la finalidad de fortalecer las publicaciones en revistas integradas en estas bases comprensivas, lo que permite mayor visibilidad e impacto de las publicaciones, mejora la clasificación de los investigadores, grupos, centros e institutos de investigación, promueve la identificación de pares académicos en otras partes del mundo y mejora la gestión de recursos para la investigación. 


\section{Ficha técnica}

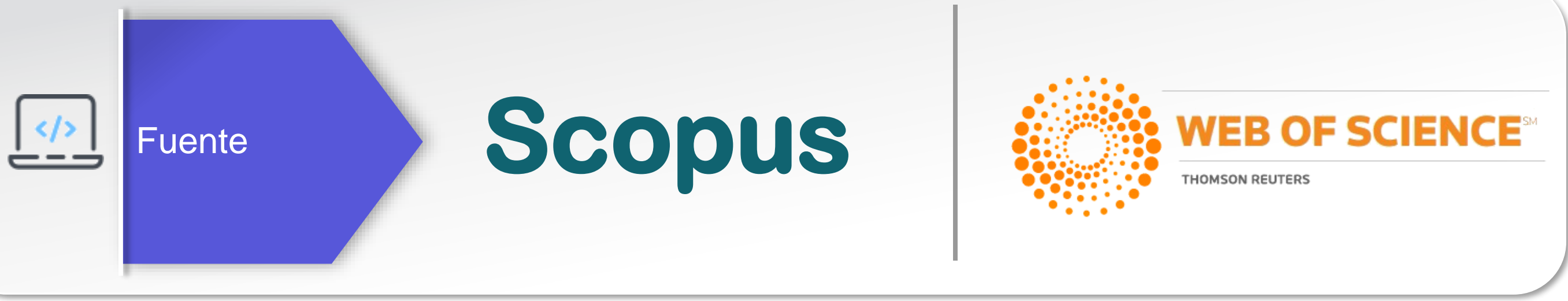

( AFFILORG ( "universidad santo tomás" OR "universidad santo tomas" OR "saint thomas aquinas university" OR "saint thomas university" OR "santo tomás university" OR "santo tomas university") ) AND ( LIMIT-TO ( PUBYEAR , 2018 ) OR LIMIT-TO ( PUBYEAR , 2017 ) OR LIMIT-TO ( PUBYEAR, 2016 ) OR LIMIT-TO ( PUBYEAR, 2015 ) OR LIMITTO ( PUBYEAR , 2014 ) OR LIMIT-TO ( PUBYEAR, 2013 ) OR LIMIT-TO ( PUBYEAR, 2012 ) OR LIMIT-TO ( PUBYEAR, 2011) OR LIMIT-TO ( PUBYEAR, 2010 ) OR LIMITTO ( PUBYEAR, 2009 ) OR LIMIT-TO ( PUBYEAR, 2008 ) OR LIMIT-TO ( PUBYEAR, 2007 ) OR LIMIT-TO (PUBYEAR, 2006 ) OR LIMIT-TO (PUBYEAR, 2005 ) OR LIMITTO ( PUBYEAR, 2004 ) OR EXCLUDE ( PUBYEAR, 2019 )) AND ( EXCLUDE ( AF-ID , "Universidad Santo Tomas, Santiago" 60009128 ) OR EXCLUDE ( AF-ID , "University of Santo Tomas Hospital" 60071489 ) OR EXCLUDE ( AF-ID , "St. Thomas University, Miami" 60026192 ) OR EXCLUDE ( AF-ID , "Saint Thomas University, Fredericton" 60018810 ) OR EXCLUDE ( AF-ID , "Saint Thomas University" 119159448 ) OR EXCLUDE ( AF-ID , "Universidad Santo Tomas de Oriente y Medio Día" 115480135 ) OR EXCLUDE ( AF-ID , "Santo Tomas University Hospital" 113845649 ) OR EXCLUDE ( AF-ID , "Family Health Center of Los Lagos" 114163380 ) OR EXCLUDE ( AF-ID , "Universidad Santo Tomás Talca" 120956329) ) AND ( EXCLUDE ( AF-ID , "Santo Tomas University" 112880585)) AND ( EXCLUDE ( FUND-SPONSOR , "Gemeinnützige Hertie-Stiftung" ) ) AND ( EXCLUDE ( AFFILCOUNTRY, "Philippines" ) )

$(\mathrm{OO}=$ universidad santo tomas $\mathrm{AND} \mathrm{CU}=\mathrm{COLOMBIA}) \mathrm{OR}(\mathrm{OO}=$ saint thomas aquinas univer sity AND $C U=C O L O M B I A)$ OR (OO=saint thomas university AND $C U=C O L O M B I A)$ OR $(\mathrm{OO}=$ santo tomás university $\mathrm{AND} \mathrm{CU}=\mathrm{COLOMBIA}) \mathrm{OR}(\mathrm{OO}=$ santo tomas university AND $\mathrm{CU}=\mathrm{COLOMBIA})$ OR $(\mathrm{OO}=$ Univ Santo Tomas AND CU=COLOMBIA)

Análisis: [excluyendo]: AÑOS DE PUBLICACIÓN: (2019)

Índices: SCI-EXPANDED, SSCI, A\&HCI, ESCI.

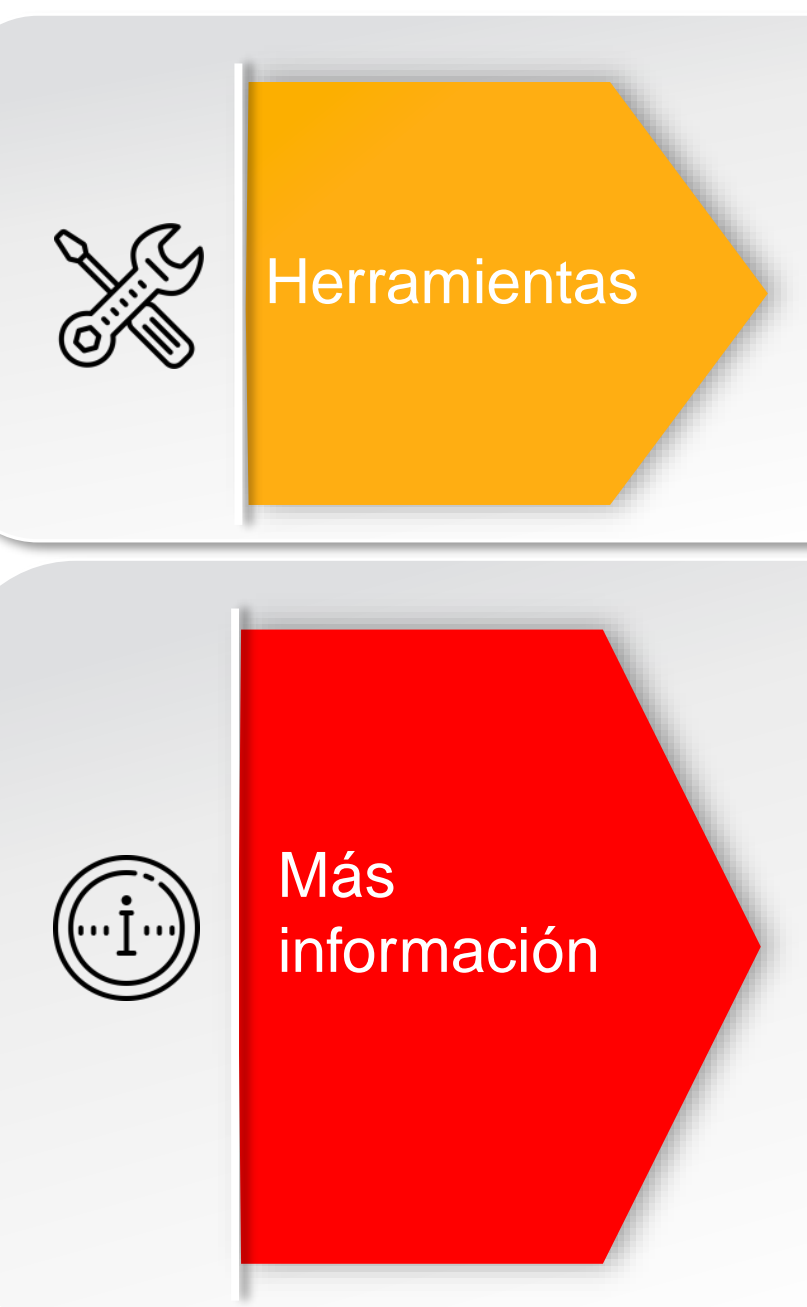

VantagePoint versión 11, Microsoft Excel, VOSviewer 1.6.10.
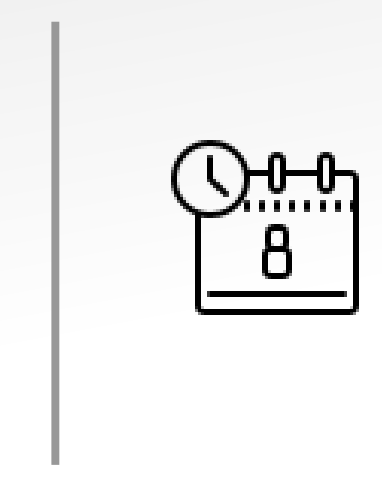

Periodo de análisis

2004-2018

\section{DATOS USTA}

http://observatoriocienciometria.usta.edu.co/index.php/metricasusta/corriente-principal

\section{DATOS ABIERTOS:}

Scopus / DOI: 10.5281/zenodo.3166896

WOS / DOI: 10.5281/zenodo.3168677

Fecha de consulta: 14/01/2019 
Metodología

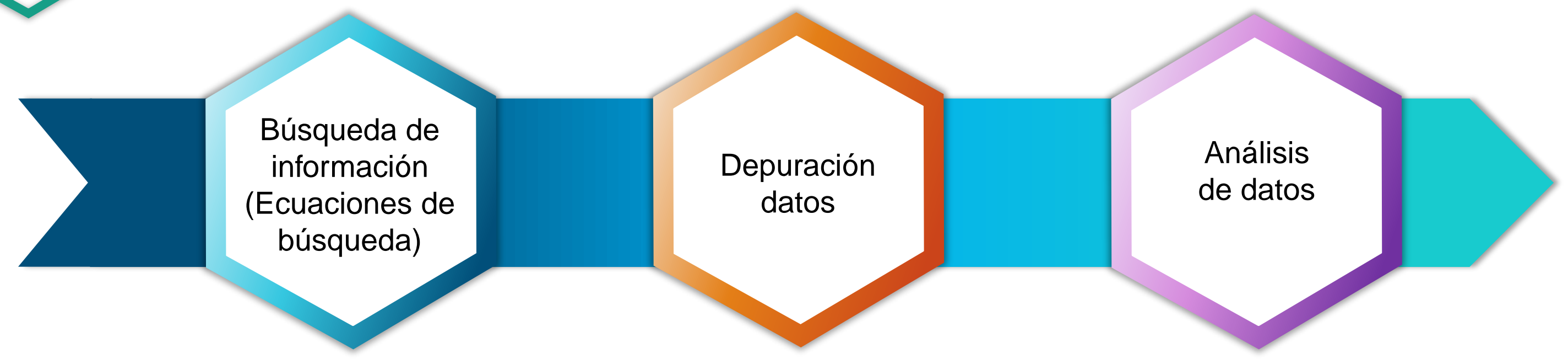

Resultados

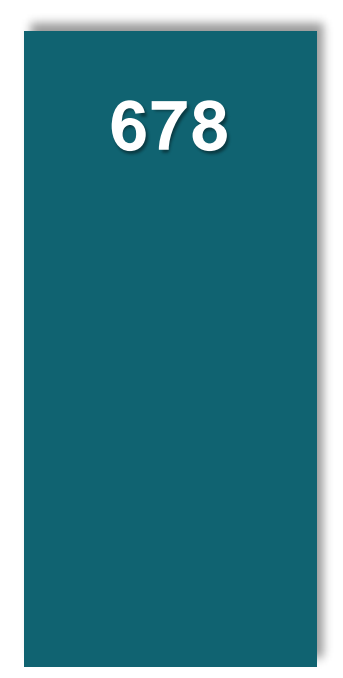

- Scopus $\square$ WOS

\section{9}

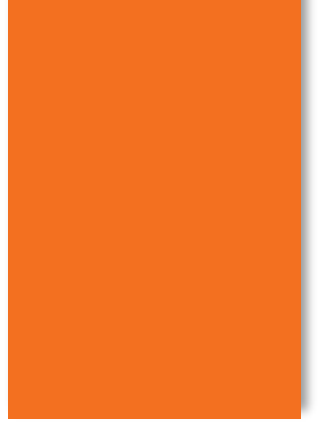

Gráfica 1. Total de publicaciones USTA en Scopus y WOS (2004-2018)

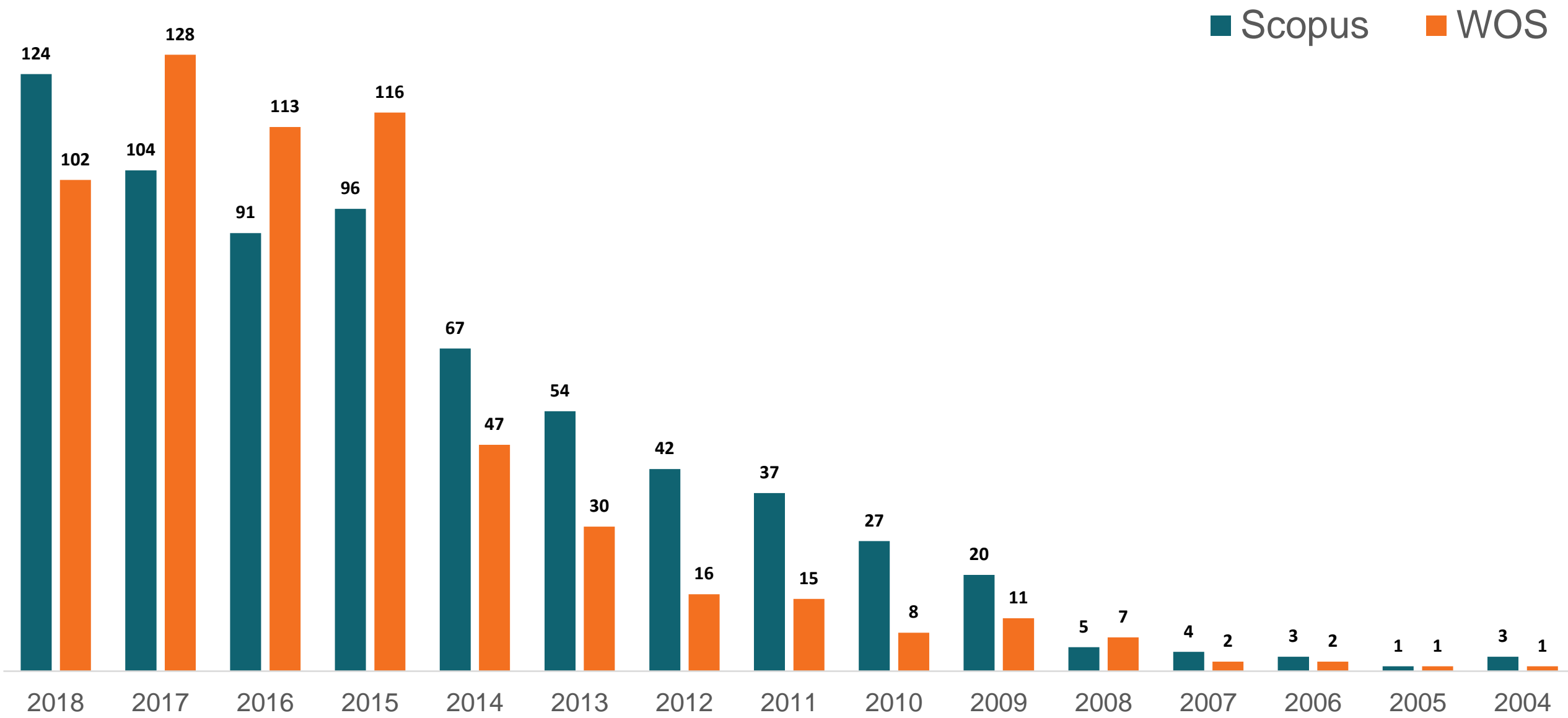

Gráfica 2. Publicaciones USTA en Scopus y WOS por año 


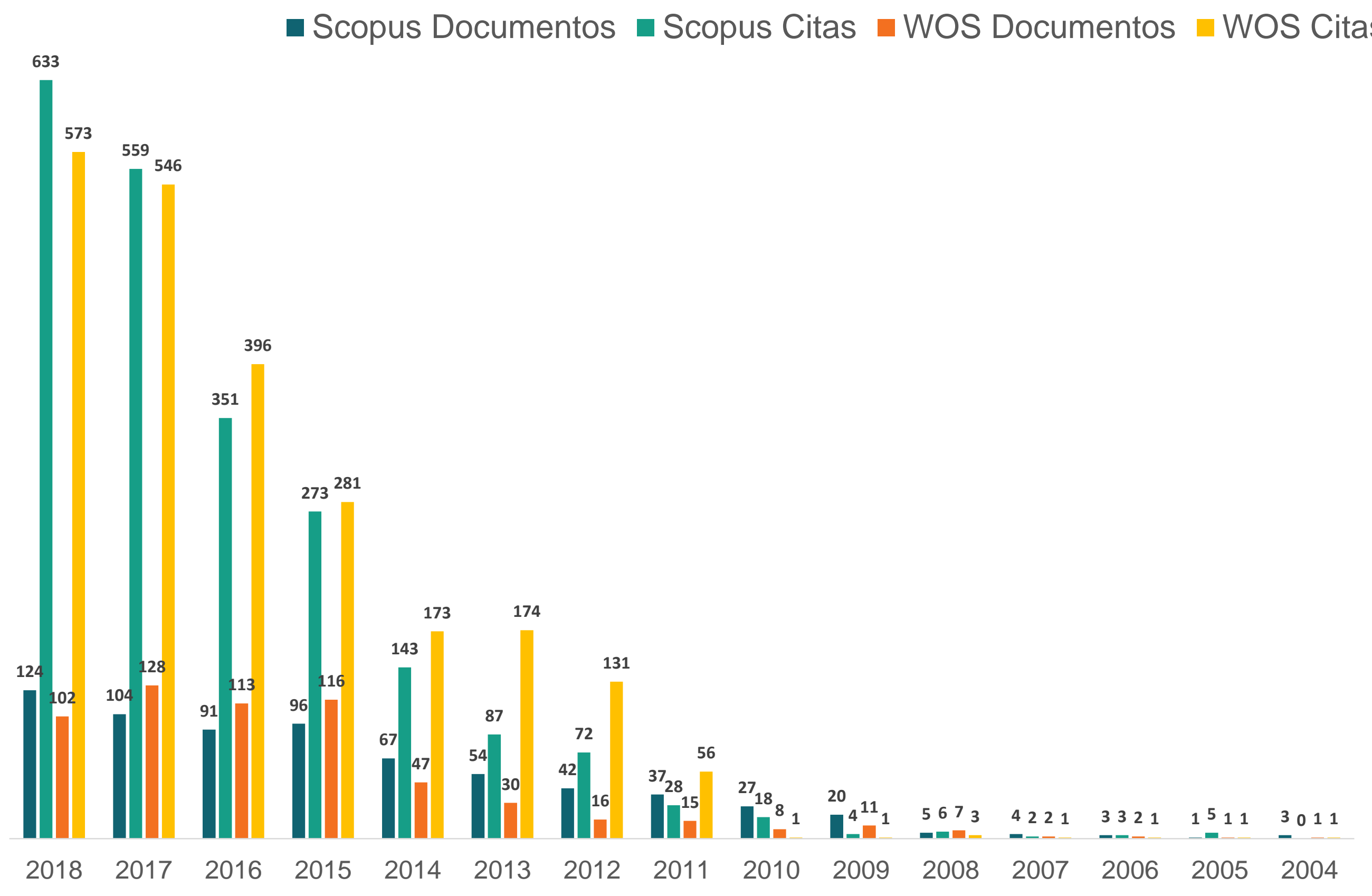

Gráfica 3. Citas y publicaciones USTA por año en Scopus Y WOS
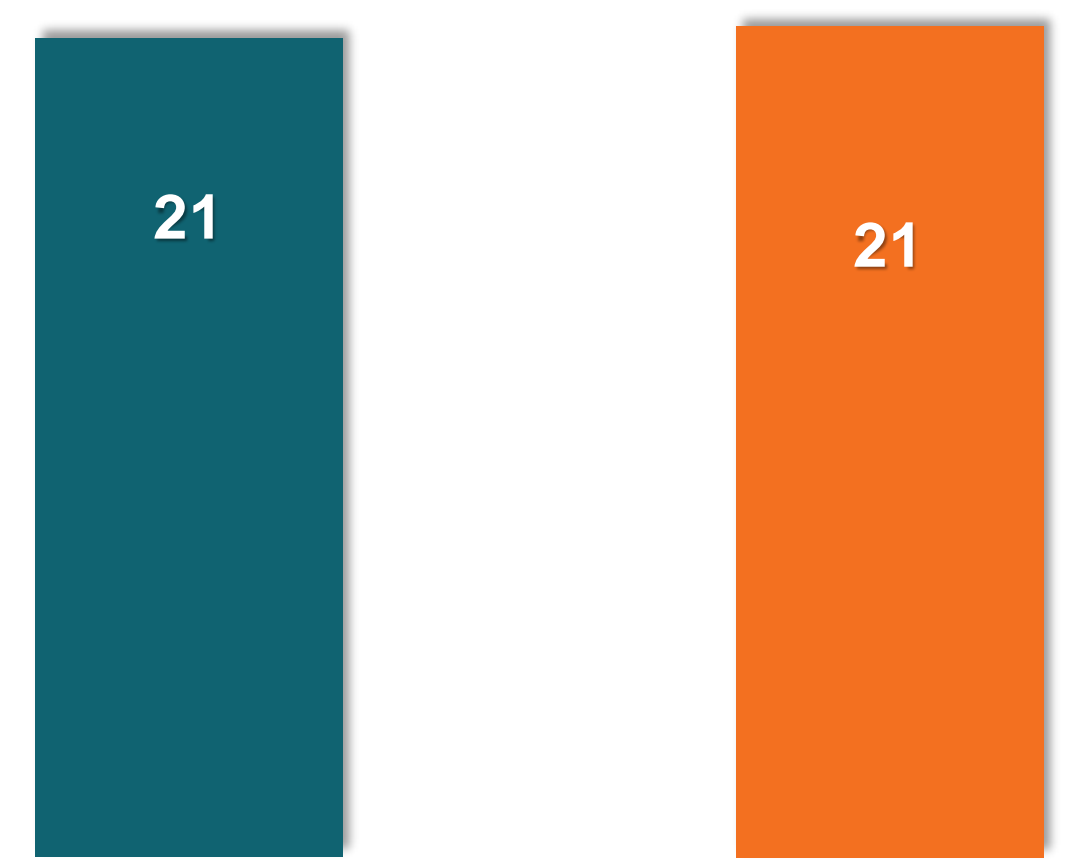

-Scopus - WOS

Gráfica 4. Índice H publicaciones USTA en Scopus Y WOS 


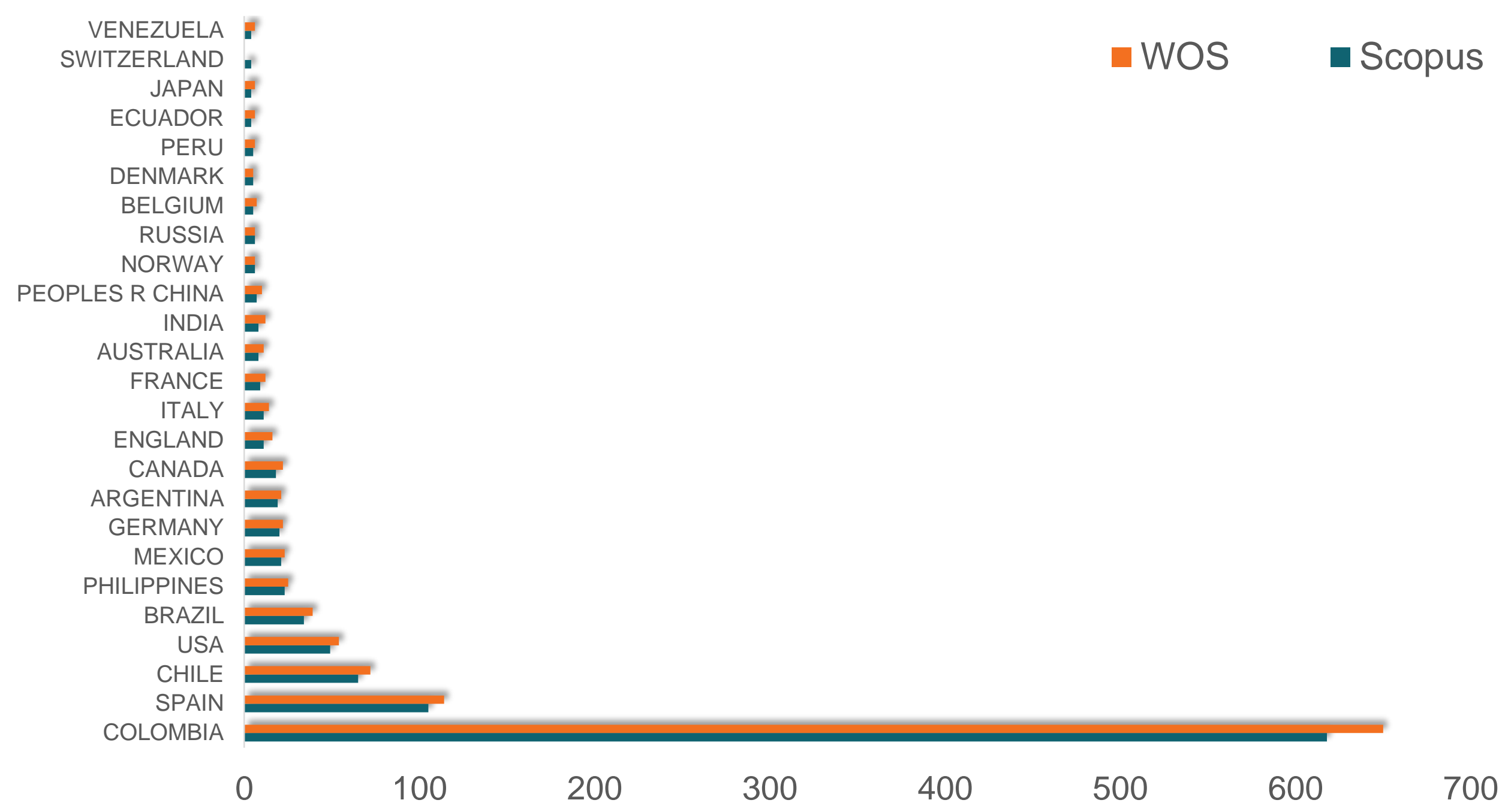

Gráfica 5. Producción USTA en Scopus y WOS por país de filiación

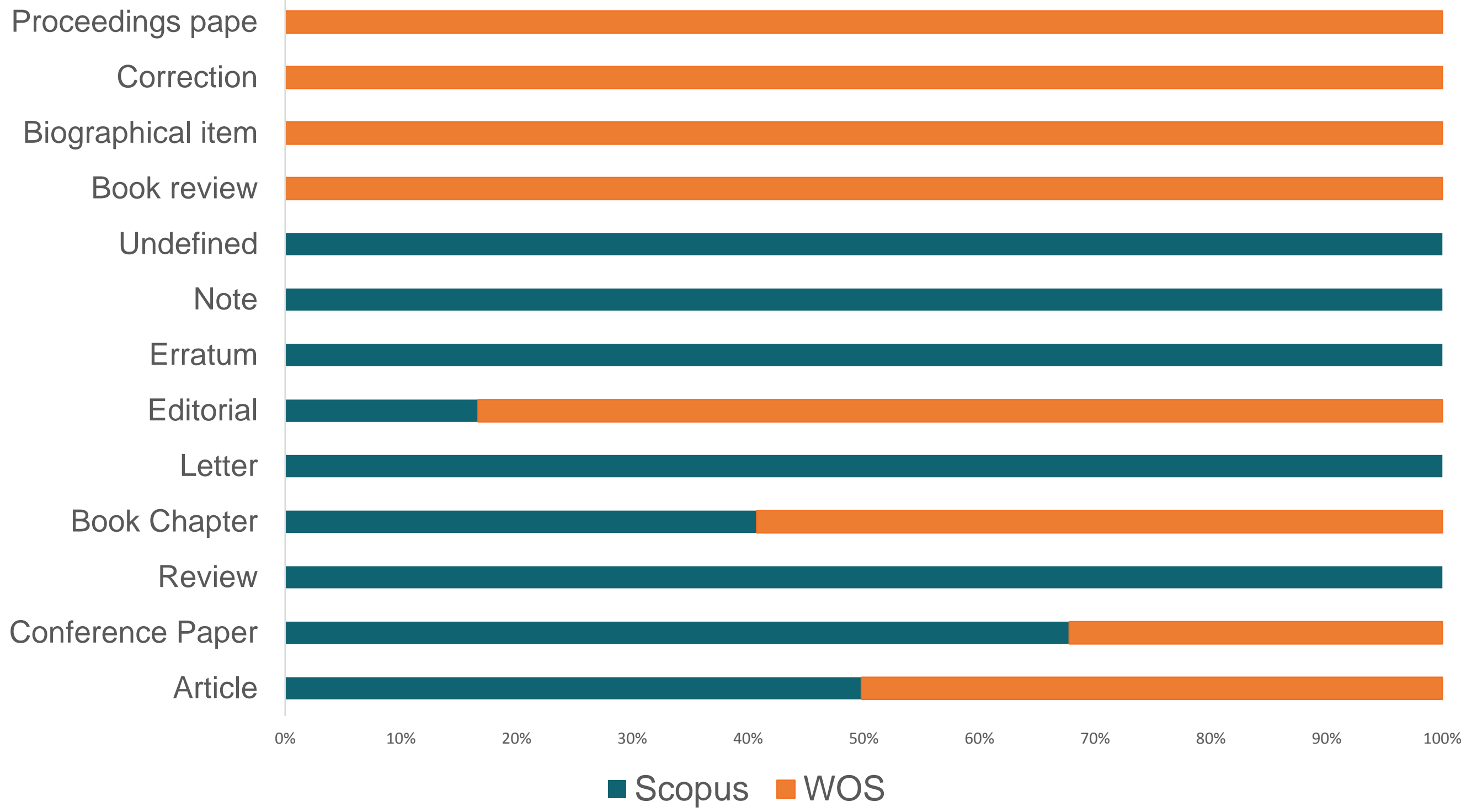

Gráfica 6. Publicaciones USTA en Scopus Y WOS por tipología documental 
Universitat Politècnica de València Universidad Autónoma de Bucaramanga

Universidad Pontificia Bolivariana Universidad de Los Andes, Colombia Universidad de Chile Universidad del Valle, Cali Universidad Pública de Navarra Universidad de La Sabana Universidad de Granada Universidad de Santiago de Chile Universitat Politècnica de Catalunya

Pontificia Universidad Javeriana Universidad Manuela Beltrán Universidad del Rosario Universidad Industrial de Santander Universidad Nacional de Colombia Universidad Santo Tomás, Bogotá $0 \%$

$20 \%$ $40 \%$ $60 \%$ $80 \%$ $100 \%$

Gráfica 7. Publicaciones USTA en Scopus Y WOS por afiliación institucional|
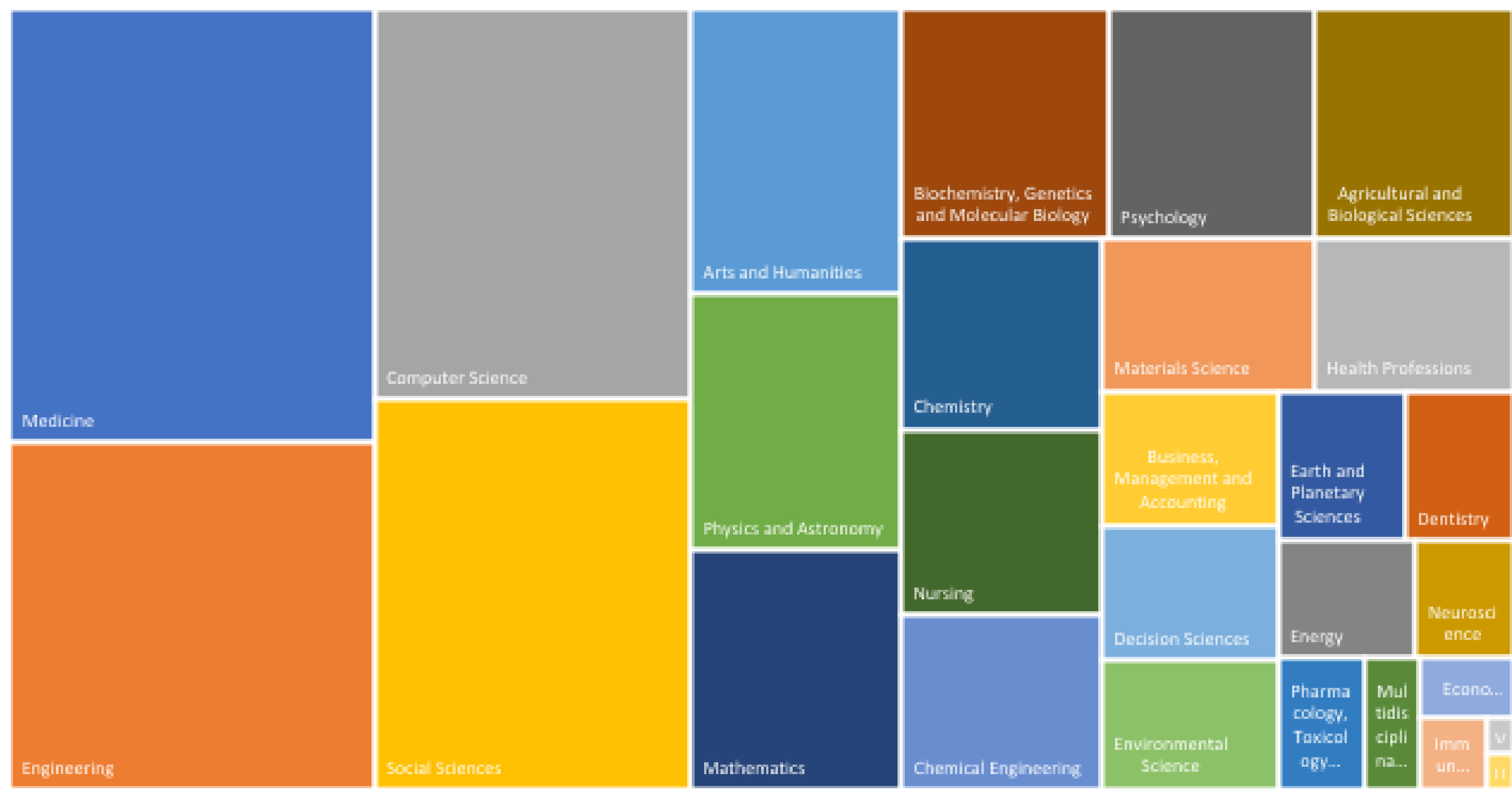

Gráfica 8. Publicaciones USTA en Scopus por áreas de conocimiento 


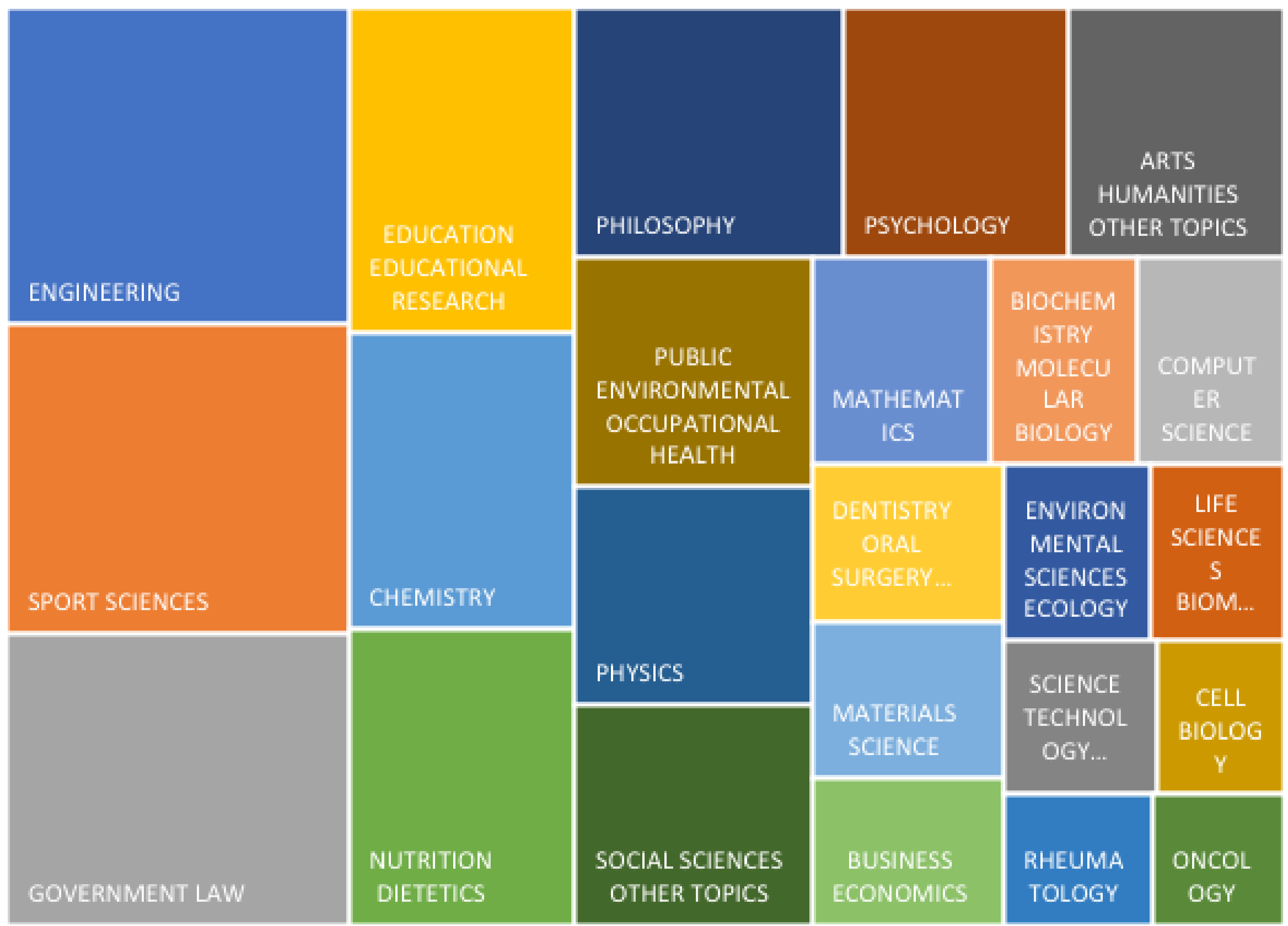

Gráfica 9. Publicaciones USTA en WOS por áreas de conocimiento

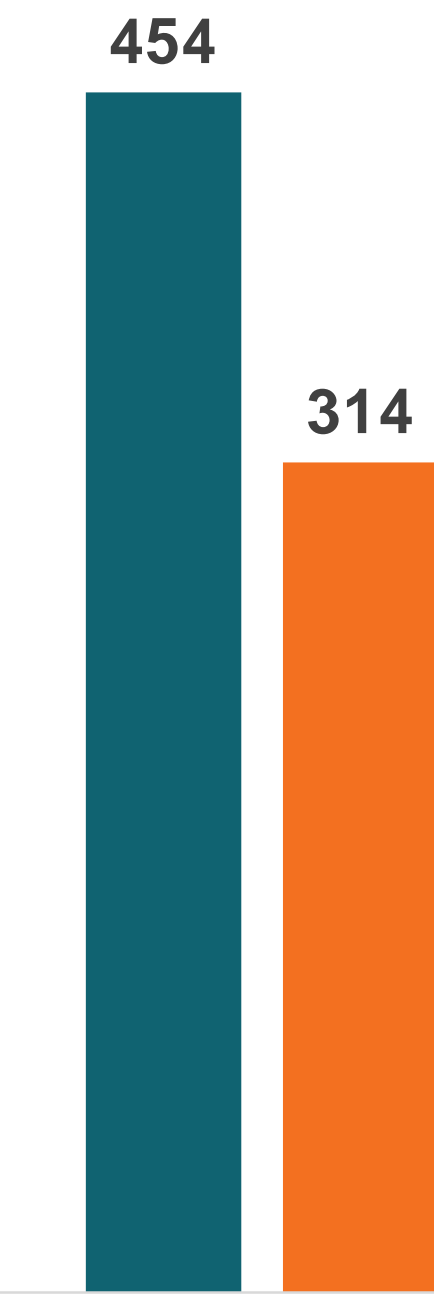

Inglés
314

283

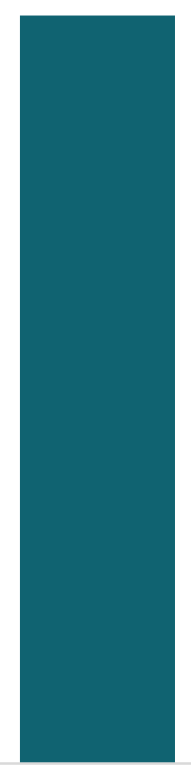

Español
28

$\frac{5}{\text { Portugues }}$

- Scopus $\quad$ WOS

Gráfica 10. Publicaciones USTA en Scopus y WOS por idioma 


\section{Top 10 publicaciones Scopus con más citas de Autores USTA}

11 Biomed Research International
2015,729076 $\begin{array}{r}\begin{array}{r}\text { Velasco, M.A., Narváez-Tovar, C.A., Garzón-Alvarado, D.A. } \\ \text { Design, materials, and mechanobiology of biodegradable } \\ \text { scaffolds for bone tissue engineering }\end{array} \\ \text { CITAS: } \\ \text { CITS: }\end{array}$

30urnal of Endodontics Alves, F.R.F., Almeida, B.M., Neves, M.A.S., (...), Rôças, I.N., Siqueira Jr., J.F.
37(4), pp. 496-501
CITAS:
ANO

4 Journal of the American Chemical Society 134(48), pp. 19607-19618

CITAS: $56 \quad$ AÑO: 2012

\section{5}

Journal of Periodontology 78(4), pp. 629-639

CITAS: $\quad 54 \quad$ AÑO: 2007

6 Surface and Coatings Technology
254, pp. 104-111
$\begin{array}{lll}\text { CITAS: } & 46 & \text { AÑO: } \quad 2014\end{array}$

Popov, A.A., Chen, N., Pinzón, J.R., (...), Echegoyen, L.A., Dunsch, L.

Redox-active scandium oxide cluster inside a fullerene cage: Spectroscopic, voltammetric, electron spin resonance spectroelectrochemical, and extended density functional theory study of Sc4 O2 @C80 and its ion radicals

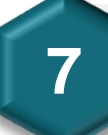
PLOS ONE 10(10),e0140875

CITAS: 42 AÑO: 2015

Lafaurie, G.I., Contreras, A., Barón, A., (...), Aya, M.D.R., Arce, R.

Demographic, clinical, and microbial aspects of chronic and aggressive periodontitis in Colombia: A multicenter study

Castillejo, F.E., Marulanda, D.M., Olaya, J.J., Alfonso, J.E.

Wear and corrosion resistance of niobium-chromium carbide coatings on AISI D2 produced through TRD

8
$\begin{array}{r}\text { Journal of Endodontics } \\ \text { 39(5), pp. 600-604 }\end{array}$$\quad \begin{aligned} & \text { Moreno, J.O., Alves, F.R.F., Gonçalves, L.S., (...), Rôças, I.N., Siqueira Jr., J.F. } \\ & \text { CITAS: }\end{aligned}$

9 Journal of Strength and Conditioning Research Ramírez-Campillo, R., Burgos, C.H., Henríquez-Olguín, C., (...), Marques, M.C., Izquierdo, M. 29(5), pp. 1317-1328

CITAS: $\quad 38 \quad$ ANO: $\quad 2015 \mid \begin{array}{r}\text { Effect of unilateral, bilateral, and combined plyometric training on } \\ \text { explosive and endurance performance of young soccer players }\end{array}$

Journal of Physiotherapy
$61(1)$, pp. 3-9

CITAS: $\quad 38$ AÑ: 2015

Meneses-Echávez, J.F., González-Jiménez, E., Ramírez-Vélez, R.

Supervised exercise reduces cancer-related fatigue: A systematic review 


\section{Top 10 publicaciones WOS con más citas de Autores USTA}

1 Journal of Endodontics

Volumen: 40 Número: 1 Páginas: 133-139

CITAS: $\quad 79$ AÑO: 2014

Becerra, Patricia; Ricucci, Domenico; Loghin, Simona; et ál.

Histologic Study of a Human Immature Permanent Premolar with Chronic Apical Abscess after Revascularization/Revitalization
BMC Cancer
Volumen: 15 Número de artículo: 77
2

62

AÑ:

2015
Francisco Meneses-Echavez, Jose; Gonzalez-Jimenez, Emilio; Ramirez-Velez, Robinson

Effects of supervised exercise on cancer-related fatigue in breast cancer survivors: a systematic review and meta-analysis

\section{3}
CITAS:

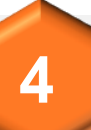

4.

CITAS: Volumen:37 Número: 4 Páginas: 496-501

Número de artículo: 729076
Velasco, Marco A.; Narvaez-Tovar, Carlos A.; Garzon-Alvarado, Diego A.

Design, Materials, and Mechanobiology of Biodegradable Scaffolds for Bone Tissue Engineering
5 Journal of strength and conditioning research Volumen: 29 Número: 7 Páginas: 1784-1795
CITAS:
52 AÑO:
2015

Journal of Periodontology

6 Volumen: 78 Número: 4 Páginas: 629-639
CITAS:
50
AÑO:
2015

\section{PLOS ONE} Volumen:10 Número:10 Número de artículo: e0140875
CITAS:

44
AÑO:
2015

Ramirez-Campillo, Rodrigo; Gallardo, Francisco; Henriquez-Olguin, Carlos; et ál.. Effect of vertical, horizontal, and combined plyometric training on explosive, balance, and endurance performance of young soccer players

Lafaurie, Gloria Ines; Contreras, Adolfo; Baron, Alexandra; et ál..

Demographic, clinical, and microbial aspects of chronic and aggessive periodontitis in Colombia: A multicenter study

Ramirez-Velez, Robinson; Rodrigues-Bezerra, Diogo; Enrique Correa-Bautista, Jorge; et ál..

Reliability of Health-Related Physical Fitness Tests among Colombian Children and Adolescents: The FUPRECOL Study

Ramirez-Campillo, Rodrigo; Burgos, Carlos H.; Henriquez-Olguin, Carlos; et ál.

Effect of unilateral, bilateral, and combined plyometric training on explosive and endurance performance of young soccer players
CITAS:
39 AÑO:
2014

Chemical Biology \& Drug Design Volumen: 82 Número: 5 Páginas: 477-499
34
AÑO:

Wear and corrosion resistance of niobium-chromium carbide coatings on AISI D2 produced through TRD

Puerto Galvis, Carlos E.; Vargas Mendez, Leonor Y.; Kouznetsov, Vladimir V.

Cantharidin-Based Small Molecules as Potential Therapeutic Agents 


\section{Top 5 autores USTA con más publicaciones}

\section{Scopus \\ WEB OF SCIENCE}

Meneses-Echavez, J. F. 33 publicaciones

Pedraza, Cesar

22 publicaciones

3 Triana-Reina, H.R.

18 publicaciones

Malagón-Romero, Dionisio H.

15 publicaciones

5 Vitola, Jaime

13 publicaciones
Meneses-Echavez J.F.

28 publicacjones

Martinez-Torres J.

23 publjcacjones

Triana-Reina H.R.

] 6 publjicacjones

Leonor Y. Vargas Méndez

10 puljolicaciones

5 publicacjones 